\title{
A comparison of the chest radiograph and computerised tomography in assessing lung changes in acute spinal injuries-an assessment of their prevalence and the accuracy of the chest X-ray compared with $\mathrm{CT}$ in their assessment
}

\author{
G Bain, R Bodley, A Jamous, S Williams and J Silver \\ Stoke Mandeville Hospital, Aylesbury, Bucks HP21 8AL, UK
}

\begin{abstract}
Lung function in patients following an acute spinal injury is frequently much more compromised than may be expected from the level of injury and the chest radiograph appearance. Experimental evidence in anaesthetised patients and subsequently our own experience with patients with acute spinal injuries suggested that in paralysed patients lung changes were frequent and that in many spinal patients large pleural effusions and lung consolidation could be present without the usual associated chest radiograph changes being recognised. This study was performed to assess the prevalence of chest pathology and the sensitivity of the chest radiograph in portraying it. Sixty patients (50 males, 10 females, 31 cervical, 29 thoracic or thoracolumbar; 15 incomplete, 45 complete; ages 17-66, mode 22 years) with spinal injuries from a variety of causes were assessed with a supine chest radiograph and three computerised tomography axial cuts at standardised locations through the thorax. The chest radiograph agreed with the computerised tomography in only 12 patients (six normal, six abnormal). The chest radiograph suggested that there were 19 normals but computerised tomography only showed 12 . In a total of 35 patients, the chest radiograph significantly underestimated the degree of change and in 13 the chest radiograph suggested greater pathology than was shown on computerised tomography. Thirteen of the 20 patients with cervical lesions but no chest trauma had lung changes. The study showed that: (1) lung pathology is a frequent sequel of a spinal injury and may be unrelated to thoracic injury; (2) the supine chest radiograph is an unreliable estimator of lung and pleural pathology in the acute stage of spinal injury; and (3) limited computerised tomography is not necessary in all cases, but if there are unexplained pulmonary complications it may reveal unexpected effusions that may require drainage or consolidation requiring specific treatment.
\end{abstract}

Keywords: acute spinal injuries; chest pathology; chest radiograph; computerised tomography

\begin{abstract}
Introduction
Immediately after an acute spinal injury there is a high morbidity from pulmonary complications ${ }^{1}$ and they are now the most common cause of death in both the acute and the chronic phases. ${ }^{2}$ This is reflected initially by hypoxia but serial chest radiographs, the most commonly used method of objectively monitoring the chest, frequently fail to elucidate the pulmonary changes. It was, therefore, initially surprising that areas of atalectasis and collapse were found at postmortem. ${ }^{3}$ More recently, pleural effusions and lung consolidation were found by one of the authors during both ultrasound scanning of the subphrenic regions in patients with a pyrexia of unknown origin and also when scrutinising computerised tomography (CT) scans through this
\end{abstract}

Correspondence: Dr R Bodley

This paper was presented as a poster at IMSOP, Ghent, 1993 area, carried out as part of the spinal assessment, for changes other than to the spine itself.

Experimental work by Hedenstierna's group in normal patients undergoing general anaesthesia for routine surgery has shown that reversible atelectasis and pleural effusions in dependent parts of the lung, which can be readily demonstrated on CT, are a frequent occurrence in those patients who were paralysed as part of the anaesthetic procedure. ${ }^{4}$

It seemed that the patient with an acute spinal injury reproduced this situation and that indeed, in many acutely injured patients, quite large pleural effusions and/or consolidation may be present without the usually sought-for chest radiograph changes being readily apparent. The experimental method was adapted to the acute injury situation and a prospective comparison made between the chest radiograph and the CT (1) to assess the prevalence of changes; (2) to assess the relative accuracy of the chest radiograph and 
CT using the CT as the gold standard; and (3) to increase our skill in interpreting chest radiographs.

\section{Methods}

Patients suitable for the study (those with cervical and thoracic injuries) were examined on the CT scanner (in skull traction if appropriate) through the fracture level and, in addition, three further chest cuts were taken if they were not already included in the field. These were (1) at the level of the right hemidiaphragm; (2) $5 \mathrm{~cm}$ above this level; and (3) through the arch of the aorta (Figure 1).

The chest radiographs were assessed 'blind' for the presence of lung pathology by a physician and a radiologist and the results correlated with the CT cuts which were taken as the 'gold standard' because they give a very clear and accurate three dimensional idea of pleural and parenchymal changes without the superimposition inherent in plain film radiography. ${ }^{5}$

The exercise was performed twice with random presentation of the unmarked films and correlation made after each viewing. The changes were classified as follows.

Chest radiograph (Figure 2)

- Small effusion-apical cap only or $<2 \mathrm{~mm}$ lateral fluid thickness

- Medium effusion - apical cap and/or 2-7 mm lateral fluid thickness

- Large effusion-apical cap and/or $>7 \mathrm{~mm}$ lateral fluid thickness

$C T$ (Figure 3)

- Small effusion $-<5 \mathrm{~mm}$ fluid thickness

- Medium effusion-fluid level up to the level of the posterior longitudinal ligament (PLL)

- Large effusion-fluid level above the level of the PLL

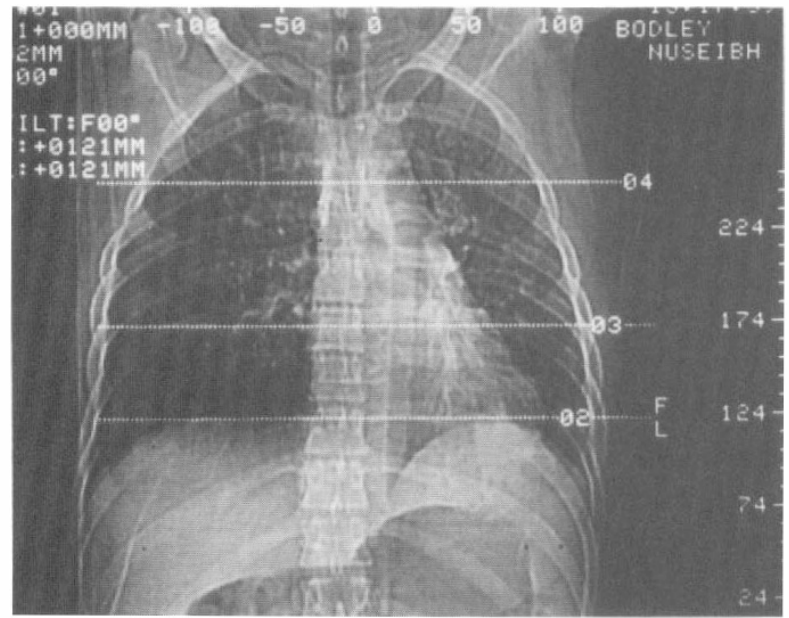

Figure 1 Scout view of the CT cuts through the thorax

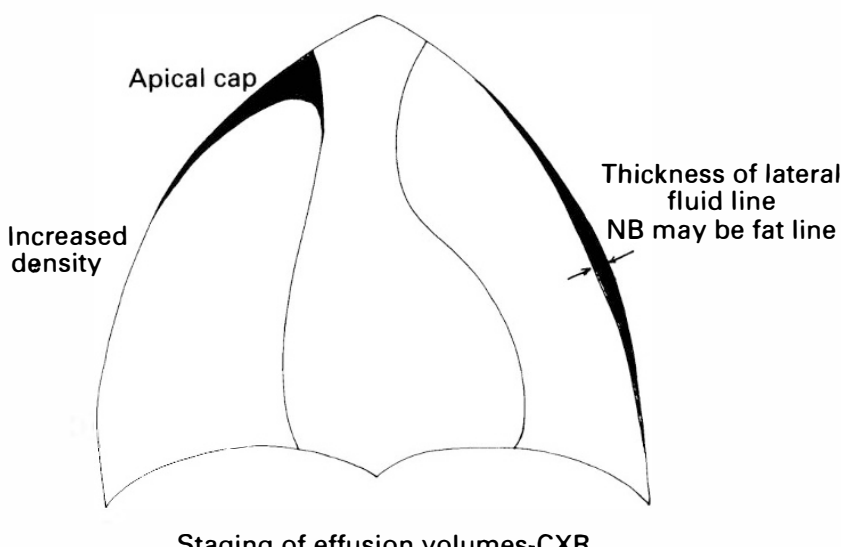

Figure 2 Schematic diagram of the changes noted on the CXR

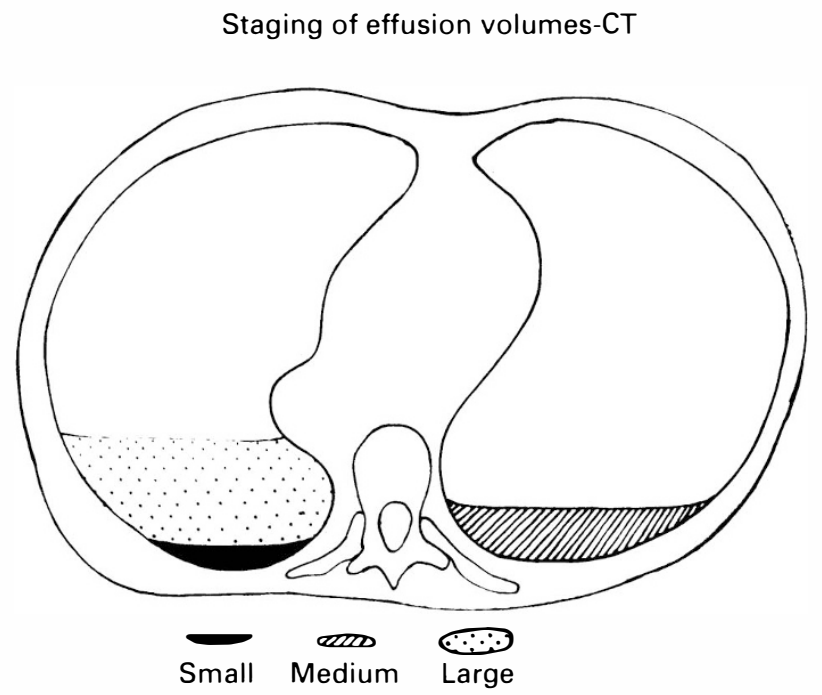

Figure 3 Schematic diagram of the distribution of pleural fluid in the supine patient as seen by CT

\section{Patients}

Between December 1985 and July 1992, 60 patients had suitable investigations for the study. There were 50 males and 10 females aged 17-66, mode 22 years. Thirty-one had cervical and 29 thoracic or thoracolumbar fractures; 15 had incomplete and 45 complete lesions. The investigations were performed mostly within 2 weeks of injury.

\section{Results}

The results are summarised in Table 1.

There was a slight improvement in accuracy on the second viewing, with both physician and radiologist improving their correlation scores between the chest radiograph and CT. Taking the results of the second viewing, there was agreement of interpretation between the chest radiograph and CT on only 12 of the 60 
Table 1 Summary of results

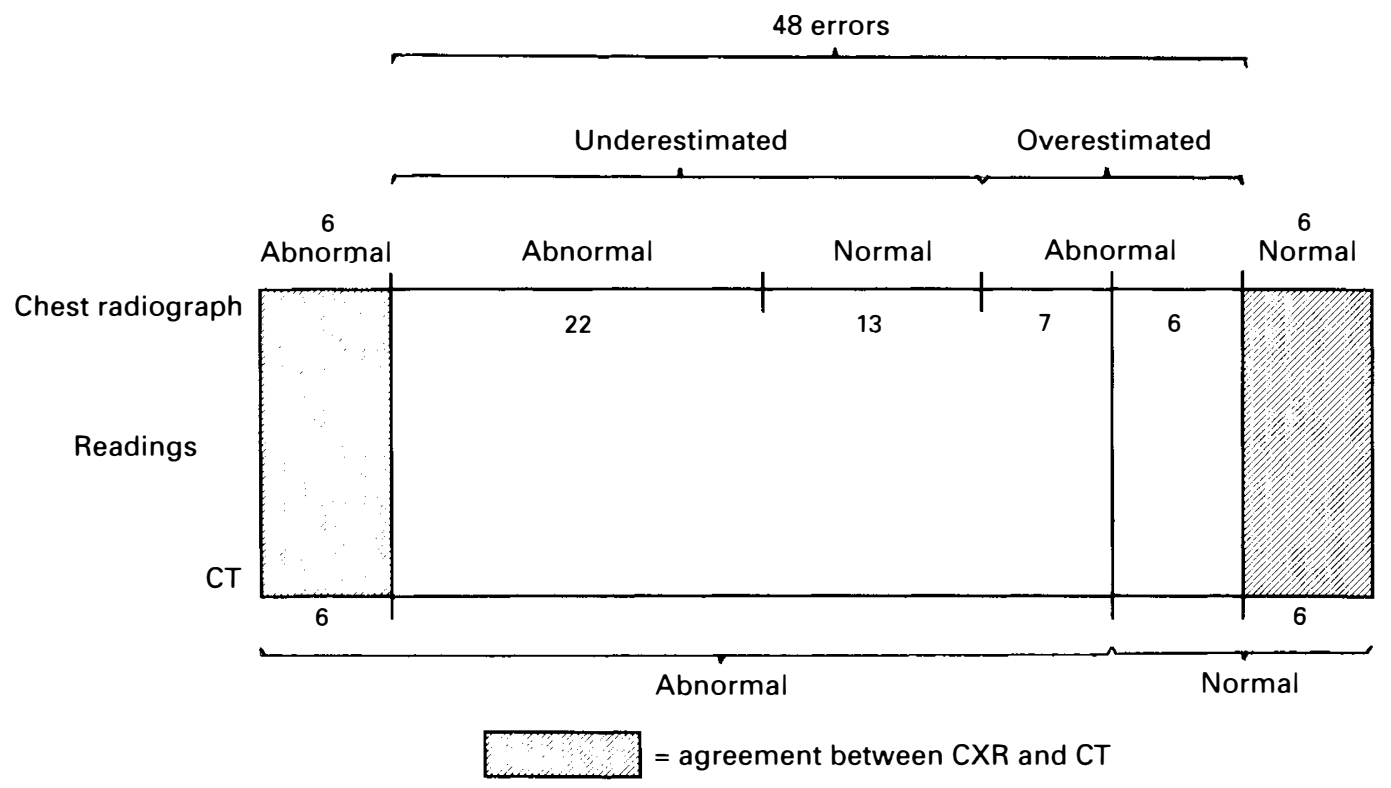

patients (six normal and six abnormal). In 35 of the 60 , the chest radiograph interpretation underestimated the changes demonstrated on CT and in another 13 they were overestimated.

The chest radiograph suggested that 19 of the 60 patients were normal but the CT showed that in fact only 12 were normal. Of these however, six had been interpreted as abnormal on the chest radiograph. A further seven chest radiographs were interpreted as showing greater abnormalities than could be seen on CT; ie 13 of the 60 chest radiographs were 'over-read'.

Thirteen of the patients with a 'normal' chest radiograph had abnormalities on CT and in a further 22 the CXR had underestimated the extent of changes shown on the CT making a total of 35 chest radiographs that were 'under-read'.

Thirteen of the 20 patients with cervical lesions but no chest trauma had changes on CT and two of the 29 patients with thoracic injuries had no significant changes in the lungs, indicating that this is not a result of direct trauma.

\section{Discussion}

Patients with spinal injuries often suffer multiple trauma and the supine chest radiograph is mandatory during early management. The supine chest radiograph is, however, significantly more difficult to interpret than the standard erect chest radiograph and there has always been a suspicion that significant pathology is frequently unrecognised. Previous advice to use the lateral chest radiograph ${ }^{3}$ is rarely followed today in most centres.

CT allows a more accurate assessment than does the chest radiograph of changes in the lung, the pleura and the surrounding bones and soft tissues, so is currently the imaging 'gold standard' for this area. Plain tomo- graphy is insufficiently sensitive and cannot be performed in the useful axial plane. MR currently does not provide a good assessment of lungs because of movement problems but may develop into a very useful technique with new fast sequences and an increasing availability. The work of Lindber et al on normal patients undergoing anaesthesia for abdominal surgery showed that they developed pleural effusions and atelectasis in the dependent lung which could be very clearly demonstrated on CT but not on a chest radiograph. We thought that a similar process might be occurring in patients with acute spinal injuries, even though they had not sustained any overt chest damage. The idea is not new and in 1967 Silver published a study of the lung complications of 50 consecutive acute spinally injured patients -18 patients developed pneumonia as demonstrated on serial lateral horizontal beam chest radiographs (to show the posterior parts of the lung better) although they had no overt symptoms of lung infection. Of these, 17 were high lesions with paralysed chest or abdominal muscles and hence had a mechanical cause for underventilation. The problem would appear to be a difficulty in clearing secretions leading to subsequent consolidations, infections and effusions.

We had already suspected a higher than expected prevalance of changes in acutely injured patients as a result of ultrasound scanning of the subphrenic regions and from CT of the thoracic spine to assess the extent of local trauma.

Using CT as a 'gold standard' the study revealed that the supine chest radiograph is a very unreliable diagnostic tool even in experienced hands. This is not really surprising, considering the anatomy of the thorax and the behaviour of fluid in a patient in different positions. Approximately $50-100 \mathrm{ml}$ pleural fluid are needed before it is suspected on an erect lateral chest 
radiograph, where it is seen in the posterior costophrenic angle and up to $500 \mathrm{ml}$ may be present before being visible on the erect frontal chest radiograph as it is lost in the inferior gutter around the dome of the diaphragm. Lamellar effusions at the lung borders are rare as most of the fluid is free flowing and lies in the most dependent area (Figure 3).

In the supine patient, this fluid will therefore lie posteriorly with a distribution dependent on the exact degree of recumbency and only be evident as an area of diffusely increased density in the middle of the lung field (Figures 4 and 5) unless it is large enough - at least $1 \mathrm{~L}$ - to be visible climbing the chest wall laterally, or more commonly, to displace the apex of the lung caudally giving the appearances of an apical fluid cap. When the effusion is very large, the appearance is similar to that of a pneumothorax but with white instead of black shadowing (Figure 6). In some obese patients, deposition of fat under the parietal pleura in the chest wall may give the impression of an effusion. This probably caused several of the overinterpretations of the chest radiograph as the CT in these patients showed such a small or absent effusion that there could
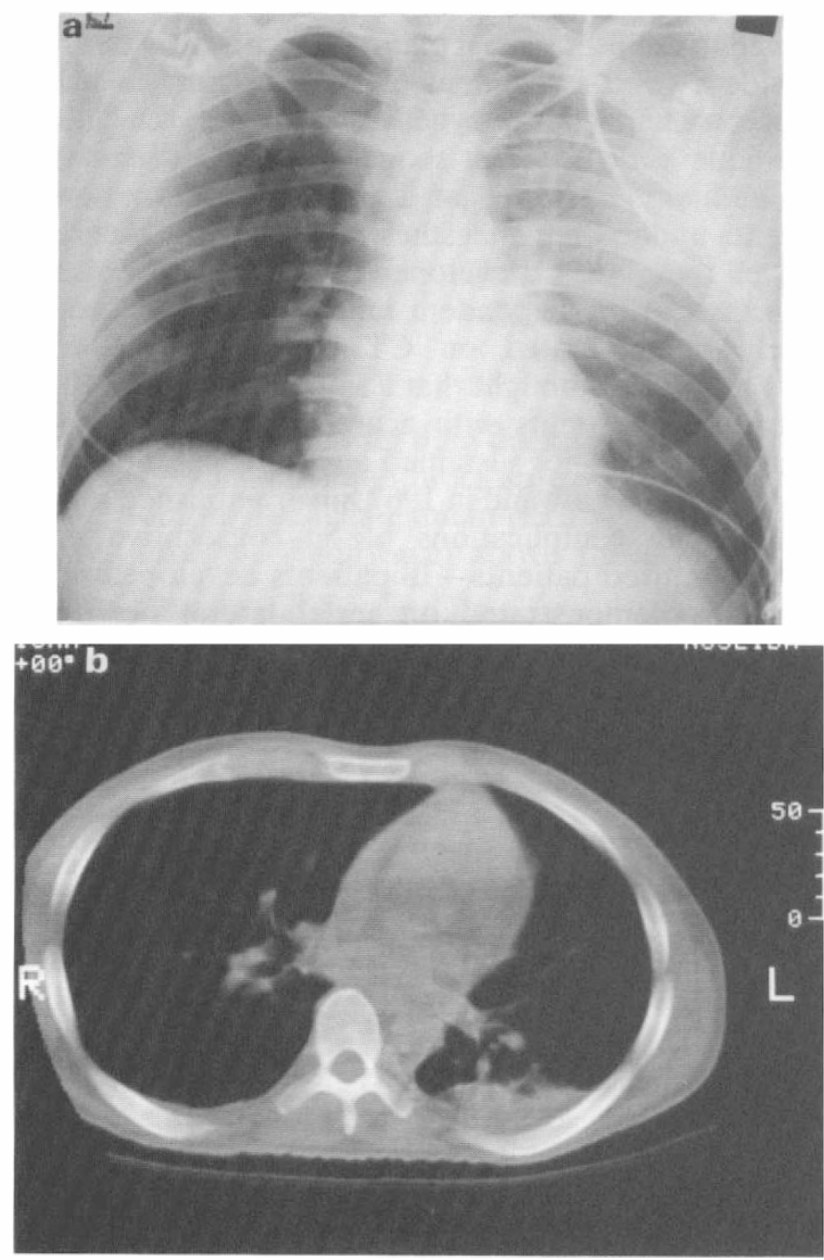

Figure 4 Unilateral increased density in the left lung field from a large effusion in a supine patient. (a) The CXR changes. (b) The same patient on CT not have been enough fluid present to displace the lung medially. Similarly, apical pleural thickening from fibrotic change is not an uncommon finding in older patients and could also have contributed to overreading. When the fluid volume is large enough to reach the hemidiaphragm, the normally clear outline becomes rather hazy (Figure 7).

The commonest under-reading error was failing to appreciate the increased opacity of the lung field caused by the fluid lying posteriorly. If the changes were bilateral then the asymmetry relied on for the diagnosis is much reduced and suspicion decreased.

The study shows that although the chest radiograph is inaccurate at assessing changes, there are certain signs that should be sought carefully (Table 2) and that practice and review increases the accuracy rate of

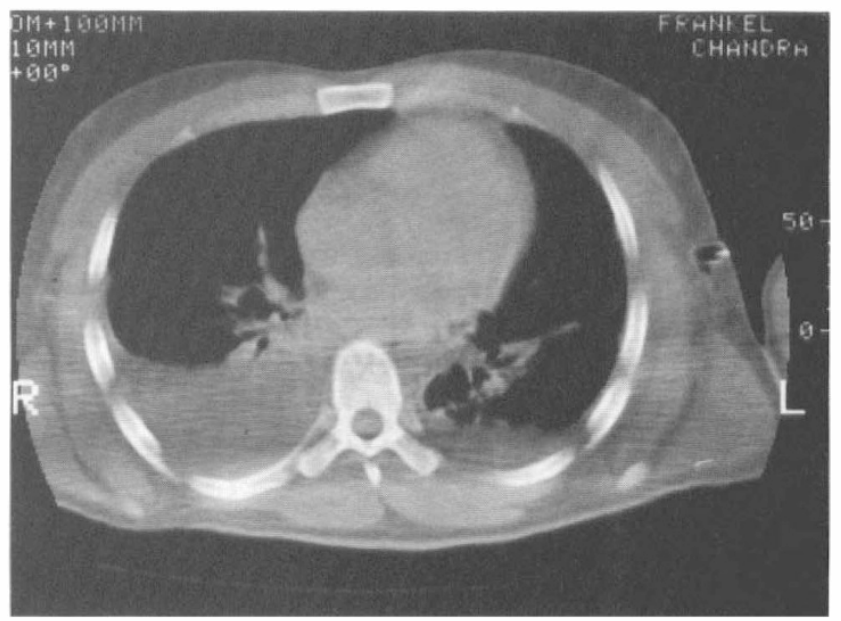

Figure 5 CT of a patient with a 'normal' chest radiograph. Note that the effusions are bilateral so that comparison with the other side is not as helpful

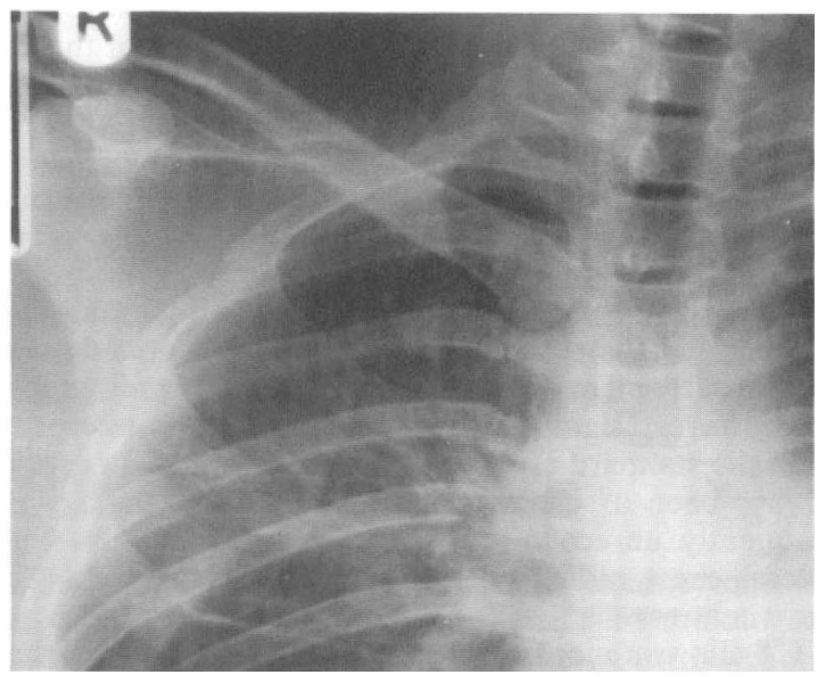

Figure 6 Chest radiograph appearances of the apical pleural cap effusion 


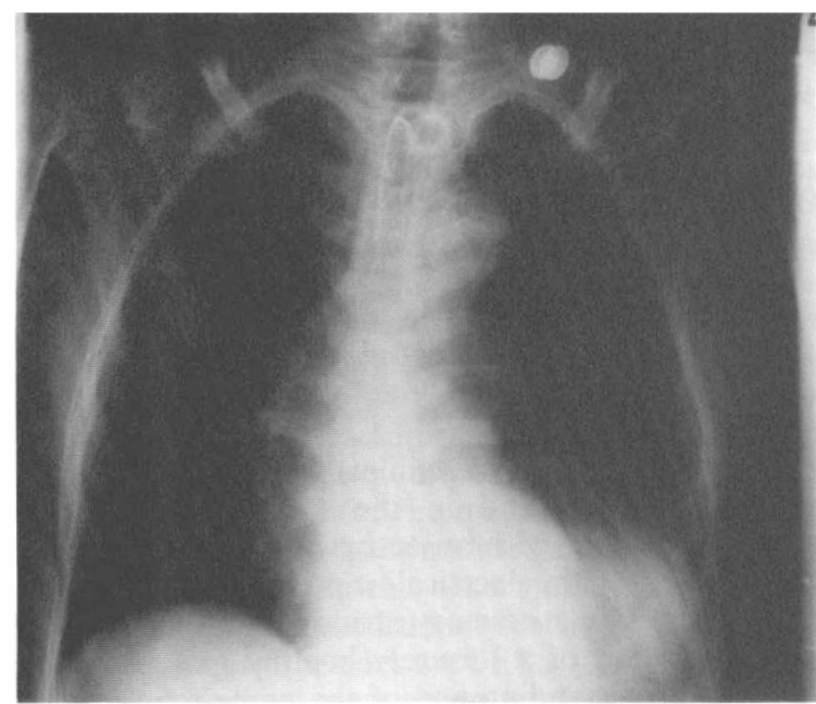

Figure 7 Chest radiograph showing a hazy left hemidiaphragm

Table 2 The most useful chest radiograph signs

(1) Apical fluid cap

(2) Hazy or indistinct hemi-diaphragm

(3) Unilateral increased lung field density

NB: The classic 'meniscus' sign of a pleural effusion is only seen on the erect chest radiograph

interpretation. It also shows that if there is still clinical concern with chest problems, a limited CT should be performed. Ultrasound would detect effusions but not the lung consolidation so accurately. In our series, only two patients had their management altered significantly by the findings - both had large haemothoraces drained with significant relief-but for the remainder of the patients the clinical picture had been clarified or confirmed.

\section{Conclusions}

(1) Pleural and pulmonary pathology is a frequent sequel of spinal injuries and may occur without clinical thoracic spine injury. It is however not an inevitable sequel to thoracic spine injury.

(2) The spine chest radiograph is an unreliable estimator of lung and pleural pathology in the acute stage of spinal injury.

(3) Limited CT is not necessary in all cases, but if there are unexplained pulmonary complications it may reveal unexpected effusions that may require drainage.

\section{Acknowledgement}

Thanks are due to Mrs Lesley Holliday for secretarial assistance.

\section{References}

1 Silver J, Morris W, Otfinowski J. Associated injuries in patients with spinal injury. Injury 1980/81; 12: 219-224.

2 Stover S. The Donald Munroe Lecture. J Am Paraplegia Soc 1994; 17: 1-6.

3 Silver J. Chest injuries and complications in the early stages of spinal cord injury. Paraplegia 1967; 5: 226-245.

4 Lindber P et al. Acta Anaesthesiol Scand 1992; 36: 546-553.

5 Flower CDR, Armstrong P. In: Grainger R, Allison D (eds). Diagnostic Radiology, Vol. 1. Churchill Livingstone: Edinburgh, 1986, p 133. 